\begin{tabular}{|c|l|}
\hline Title & Magnetic double network composite capable of large recoverable deformation \\
\hline Author(s) & Feng, Xiangchao; Ma, Zhuo; MacA rthur, Jonathan V.; Hong, Wei \\
\hline Citation & $\begin{array}{l}\text { Soft matter, 17/3), 554-562 } \\
\text { https://doi.org/10.1039/dOsm01613f }\end{array}$ \\
\hline Issue Date & 2021-01-21 \\
\hline Doc URL & http://hdl.handle.net/2115/83860 \\
\hline Type & article (author version) \\
\hline File Information & Magnetic DN Composite 11 2 final.pdf \\
\hline
\end{tabular}

Instructions for use 


\title{
Magnetic double-network composite capable of large recoverable deformation
}

Received 00th January 20xx, Accepted 00th January 20xx

DOI: $10.1039 / \times 0 \times x 00000 x$

\author{
Xiangchao Feng, ${ }^{\text {ab+ }}$ Zhuo Ma, ${ }^{\text {bct }}$ Jonathan V. MacArthur ${ }^{\text {bd }}$ and Wei Hong*efb
}

This paper presents the design and fabrication of a magnetic double network (DN) composite, which consists of permanent magnet chains embedded in an elastomer matrix, and was capable of large yet fully recoverable deformation. The initially connected magnets served as reusable sacrificial components in the composite. The strong magnetic attraction between neighboring magnetics endowed the composite with the high strength while the compliance of the elastomer matrix provided the high extensibility. Having a similar mechanism as DN gels, the composite was found to be significantly tougher than either of the constituents. The nonlinear behavior in the composite separated it into two coexisting phases - a softer phase with separated magnet links and a stiffer phase with connected magnet links - which led to the stress plateau on the tensile curve. Further stretching was manifested by the growth of the disconnected softer phase at the expense of the linked stiffer phase, until all magnets were separated. The unloading curves appeared drastically different from the loading curves, as the force needed to separate two magnets was much higher than the force at which two separated magnets snapped back. Such asymmetry between loading and unloading was the main cause of the hysteresis in the stress-strain curve and the energy dissipation. To further understand the physical mechanism and the damage process of the magnetic DN composite, a simple model was developed to examine the deformation and damage dissipation process of composite. With very few parameters, the model predictions agree qualitatively with the measured properties of the material, and the difference can be further reduced by accounting for the interfacial friction/adhesion, a second means of energy dissipation. With a combination of desired properties including high stretchability, self-healing, and high toughness, the magnetic DN composite is a viable candidate for various applications.

\section{Introduction}

Double-network (DN) gels and elastomers have been of great interest to researchers in the last decades for their superior mechanical properties including both high strength and high toughness. ${ }^{1-14}$ These desirable features endow DN gels and elastomers with applications in various fields. ${ }^{8-15}$ The toughness of DN gels and elastomers has been attributed to the interpenetrating network structures: the 1st network consists of relatively short and stiff chains while the 2 nd network of long and highly coiled chains. ${ }^{2}$ During loading, partial damage initiates in the 1st network. But the cracks in the 1st network are bridged by the chains of the 2nd network, which are gradually pulled out from the fragments of the 1st network to accommodate the large deformation and share the load. At appropriate compositions, further loading causes distributed damage of the 1st network in the surrounding area rather than rupturing the 2 nd network. Such a process results in a large process zone and energy is dissipated through breaking the 1st network and sliding between the two networks. Although such a toughening mechanism is, in principle, similar to the crackbridging in conventional composites ${ }^{16-18}$, DN gels and elastomers excel with unparalleled large strain-to-fracture. One

\footnotetext{
a. Qian Xuesen Laboratory of Space Technology, Beijing 100094, China.

b. Department of Aerospace Engineering, Ames, IA 50014, USA

c. Schlumberger Technology Corporation, Sugar Land, TX 77478, USA.

d. Department of Aeronautics and Astronautics, Massachusetts Institute of

Technology, Cambridge, MA, 02138, USA

e. Department of Mechanics and Aerospace Engineering Southern University of

Science and Technology Shenzhen 518055, China.

E-mail: hongw@sustech.edu.cn

f. Soft Matter GI-CoRE, Hokkaido University, Sapporo 060-0810, Japan
}

† These authors contributed equally to the paper.

Electronic Supplementary Information (ESI) available: [details of any supplementary information available should be included here]. See DOI: 10.1039/x0xx00000x may naturally wonder whether similar DN toughening mechanisms can be applied to macroscopic composite materials. As the afore-described mechanism is not limited to molecular scales, it is theoretically applicable to structures of all sizes. By using a fabric mesh in place of the 1st network, and an acrylic tape to mimic the 2nd network, a macroscopic composite has been demonstrated, 27 confirming the applicability of the DN mechanism to macroscopic structures. Other types of DN composites have also been demonstrated. ${ }^{19-}$ 21 Just like DN gels and elastomers with chemically crosslinked 1st network, ${ }^{1,6-7}$ these DN composites have reduced and irrecoverable stiffness and toughness once damaged. ${ }^{10}$

In contrast, when reversible ionic crosslinkers are used in DN gels, they exhibit self-healing capability as the partially damaged 1st network recovers by reconnecting the broken crosslinks. ${ }^{22}$ Similar behaviors have also been observed in other tough hydrogel systems such as polyampholytes and poly-ion composites. ${ }^{23-25}$ Inspired by the salient self-healing capabilities of the ionic crosslinks in the tough gels, we designed a magnetic DN composite capable of large and recoverable deformation in this work. In analogy to the reversible crosslinks in self-healing hydrogels, ${ }^{22-25}$ permanent magnets served as the reusable sacrificial components. The magnets were initially connected, forming fibers to provide the composite relatively high stiffness. Under a critical axial tension, the magnets separated from each other in sequence, and redistributed the load to the elastomeric matrix. Exhibiting a long plateau over the stress-strain curve, the damage evolution process was like that of DN gels and elastomers. The strength of the composite was limited by the magnetic attractions while the maximum stretch was limit by the stretchability of the matrix. The composite was significantly tougher than either of the constituents. Upon unloading, the magnetic attraction between neighboring magnetics held them back again and the composite's stiffness and strength were fully recovered. 
Even though the base materials chosen for this study may not be optimal, the results demonstrate the possibility of constructing novel macroscopic composites using the toughening mechanism of DN gels. Replacing the permanent magnets with electromagnets, one could develop a DN composite with adjustable toughness. By combining the properties of high stretchability, self-healing, and high toughness, the DN composite is a viable candidate for many applications. Materials or components designed under this mechanism may play a unique role in extreme environment or against natural disasters, for fast service resuming after destruction. Composites working under similar mechanisms may have potential applications in prosthetics such as tendons and artificial skins. Additionally, similar composites could possibly be used in inflatable structures for spatial and extraterrestrial applications.

It should also be noted that numerous studies have been carried out recently on various types of composites by integration of permanent magnets to modify their mechanical properties, to enable magnetic actuation, or to produce smart metamaterials. ${ }^{28-33}$ Although all these materials utilize similar constituents as the one presented in the current study, most of them do not exhibit the similar damage-distribution mechanism demonstrated herein. Several studies aim at altering the elastic properties and wave propagation characteristics of these materials,,$^{33}$ and pay less attention to the fracture behavior or toughness. The insignificant toughening effect in these materials is likely to be due to the unmet conditions, which will be discusses in the current paper. When proper structural or material parameters are taken, some of these metamaterials may also exhibit similar behaviors.

\section{Experimental}

The magnetic DN composite samples were prepared by first pouring the well-mixed Polydimethylsiloxane (PDMS, WACKER SILICONES) precursor solution into a mold, around the chains of cylindrical permanent magnets (Nickel Alloy HX) of two different sizes $(\$ 4 \mathrm{~mm} \times 10 \mathrm{~mm}$ for the bigger magnets, $\$ 2 \mathrm{~mm} \times 1 \mathrm{~mm}$ for the spacers) inside rectangular molds, as illustrated by Fig. 1 . The (smaller) spacer magnets (usually 3 in a group) are introduced to facilitate sliding and suppress cavitation at loading. Once fully cured, the composite samples were removed from the molds for testing. The mechanical tests

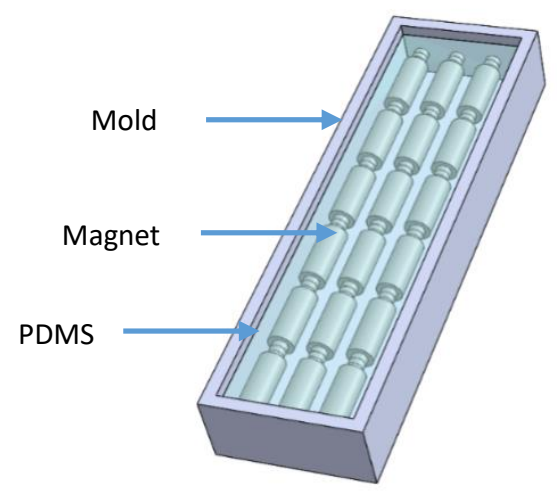

Fig. 1 Sketch of the manufacture process of the magnetic DN composite. were carried out with an Instron 5960 dual column testing system. Each sample was held vertically by metallic grippers with the magnetic chains aligned in the loading axis. After tests of identical samples at various nominal strain rates $(0.0015$, $0.003,0.006$, and $0.012 \mathrm{~s}^{-1}$ ), negligible rate dependence was found in the mechanical properties of interest. The primary loading rate used for most tests was a constant extension rate of $0.003 \mathrm{~s}^{-1}$ for repeatability and minimal noise-to-data ratio of the testing system. Each sample was loaded to up to $500 \mathrm{~mm}$ of total extension or until rupture. Additional cyclic load-unload tests at various extensions were also conducted. A video camera was used to capture the separation events of magnet links and to record the corresponding deformation processes. The composition of each sample will be noted by the arrangement of magnetic columns and number of spacer magnets used. For comparison, control samples of PDMS and magnetic columns were made into the same size and tested.

A typical force-extension curve of the magnetic DN composite of dimensions $55 \mathrm{~mm} \times 7 \mathrm{~mm} \times 7 \mathrm{~mm}$, with one single chain of magnets and $26 \%$ magnet volume fraction, subjected to uniaxial tension is shown in Fig. 2, together with the reference curves measured from a solid PDMS sample of the same dimensions and a set of magnets in the same arrangement. Compared to a chain of magnets, the composite exhibited much higher extensibility, close to that of the PDMS. The extensibility of the composite can be attributed to the damage-delocalization mechanism provided by the DN structure. Upon separation of each magnet link, large deformation was manifested locally by the PDMS, which took over the load and stabilized further damage localization. Here, the magnet chains resembled the 1st network of DN gels, while the PDMS is analogous to the 2 nd network. To accommodate the large stretch, the deformation had to be non-affine between the magnets and the matrix. Similar to those of DN gels and non-recoverable DN composites, ${ }^{2,26}$ a typical forceextension curve of the magnetic DN composite subjected to

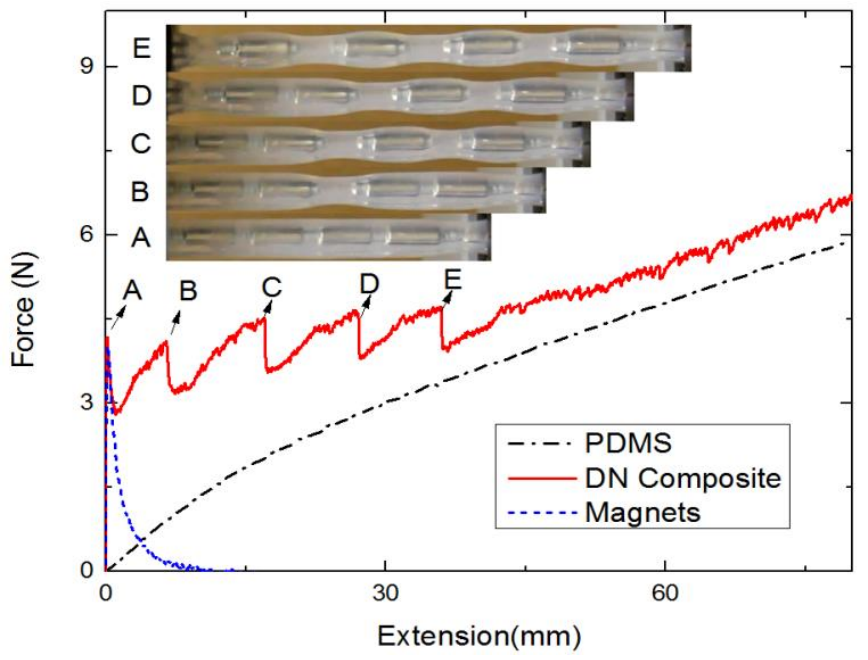

Fig. 2 Force-extension curves of a magnetic DN composite, a sample of solid PDMS with the same dimension, and a pair of magnets. The insets A-E are the snapshots of the sample corresponding to the specific points along the loading curves. Over the stress plateau (A-E), partially damaged regions coexisted with the undamaged regions, and the extension was accompanied by the growth of the damaged regions. In the strain-hardening state, the entire magnet chain was separate, and the extension caused further sliding between magnets and PDMS. 


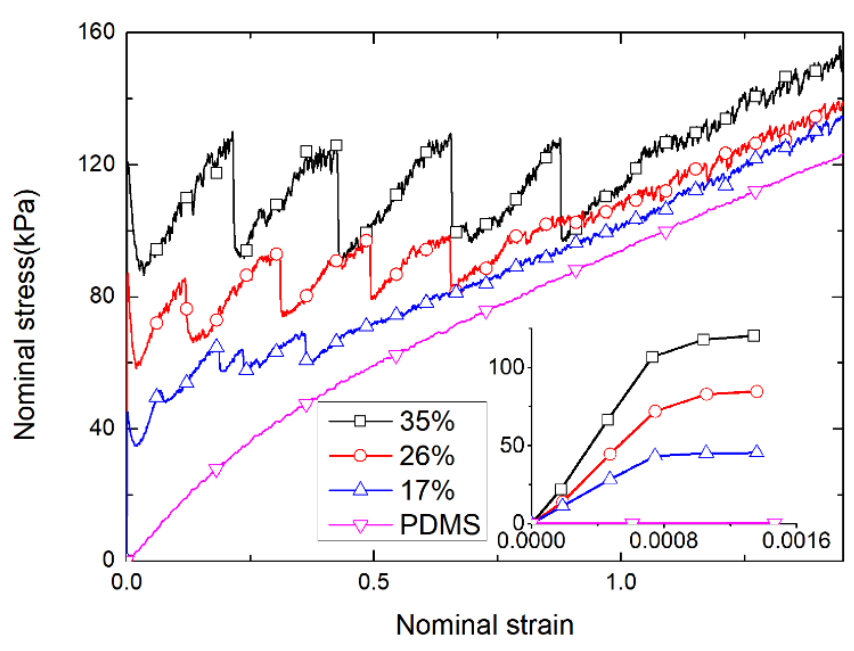

Fig. 3 Nominal stress-strain curves of the magnetic DN composite at various compositions, from $17 \%$ to $35 \%$ magnet volume fraction. The inset shows a closeup view of the initial stage.

uniaxial tension also exhibited three stages: (I) an initial stage of linear-elastic deformation, (II) a relatively flat stress plateau with sawtooth patterns accompanying the separation events of individual magnet pairs, and (III) a strain-hardening stage until final rupture. Corresponding to the stress plateau in stage II was the coexistence of two phases: a partially damaged phase with separated magnet links and an intact phase with connected magnet links, as shown by the insets of Fig. 2. No matrix (PDMS) damage was observed in either phases. This phenomenon is like the stable necking in DN hydrogels and composites. ${ }^{2,10}$ Over the stress plateau, further stretch was manifested by the growth of the partial damage zone at the expense of the intact magnetic links. Stage II continued until all the magnets were separated. In stage III, deformation was accompanied by further stretching of the matrix and sliding between the magnets and the matrix.

To study the effect of composition, samples of various magnet volume fractions were fabricated by incorporating multiple magnet chains and varying the overall dimension. Figure 3 shows the measured stress-strain curves of these samples under uniaxial tension, with the initial parts of the curves enlarged in the inset. The volume fraction of the magnets was calculated by dividing the cross-sectional area of a bigger magnet by that of the entire sample. The nominal stress was calculated by dividing the axial force by the cross-sectional area of the sample before deformation, while the nominal strain was the ratio between the axial extension and the original length. The consistency of the results was checked across several samples of the same compositions. Both the peak stress and the plateau stress increased continuously with the magnet volume fraction. At low magnet volume fractions, the performance of a DN composite was close to that of PDMS.

The hysteresis and energy dissipation of the DN composite were also studied by subject samples of various sizes to uniaxial tension with intermediate unloading at a set extension rate of $0.003 \mathrm{~s}^{-1}$. By varying the number of magnetic columns in each sample, a range of effective magnet volume fractions was tested. A representative result ( 26 vol\% magnet) is plotted in Fig.4. Without a clear stress-plateau and the sawtooth patterns

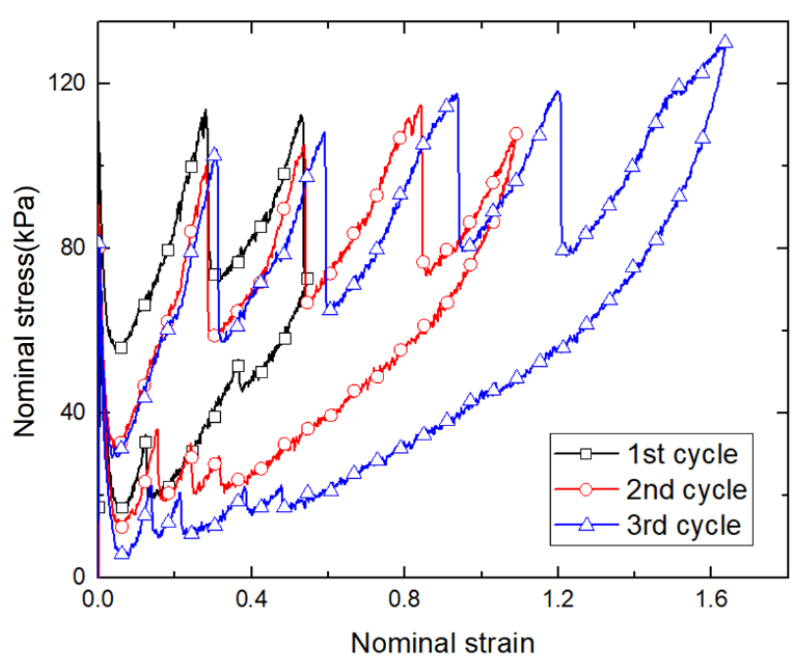

Fig. 4 Loading and unloading curves for a DN composite sample (26 vol\% magnet) subject to uniaxial tension. The small discrepancy between the 1 st and subsequent cycles is likely due to the initial bonding at the magnet-matrix interface.

took place at much lower stress and strain levels, the unloading stress-strain curves appeared drastically different from the loading curves. The loading and unloading curves enclosed large hysteresis in all cases when magnet links are separate. When reloaded, the nominal stress plateaued at approximately the same level as in previous cycles, indicating the recovery of both stiffness and strength. The small difference between loading and reloading curves might be attributed to the initial interfacial adhesion between magnets and the PDMS matrix prior to the first separation, in contrast to the friction force between the two when sliding took place. These observations will be further analyzed through a theoretical model in the following section, from which the underlying toughening mechanism shall be revealed.

\section{Theoretical}

To rationalize the toughening mechanism of DN magnetic composite, let us consider a simple 1D model for the damage distribution and energy dissipation process. The energy dissipation is through multiple levels, and we will start at the unit-cell level. Each unit cell of the composite includes two (half) magnets, the spacers in between, and the surrounding PDMS matrix, as shown by Fig. 5. In experiments, separation was only observed among the smaller spacers, so that the magnets were divided into two groups, both contain bigger magnets and smaller spacers. For simplicity, the magnetic interaction in a unit cell is modeled as that between a pair of magnetic dipoles, each representing a magnet group, and the elasticity of the polymer matrix is modeled by a spring in parallel, as sketched in the inset of Fig. 5. The sliding friction or adhesion force between them is neglected in this simple model. The model simplification allows us to decompose the nominal axial stress into two parts: the elastic nominal stress $s_{\mathrm{e}}$ and magnetic nominal stress $s_{\mathrm{m}}$.

The attractive force between two magnetic dipoles at distance $r$, with dipole moments $m_{1}$ and $m_{2}$, and directions aligned along the axis, is given by 


$$
f_{\mathrm{m}}=\frac{3 \mu_{0} m_{1} m_{2}}{2 \pi r^{4}} .
$$

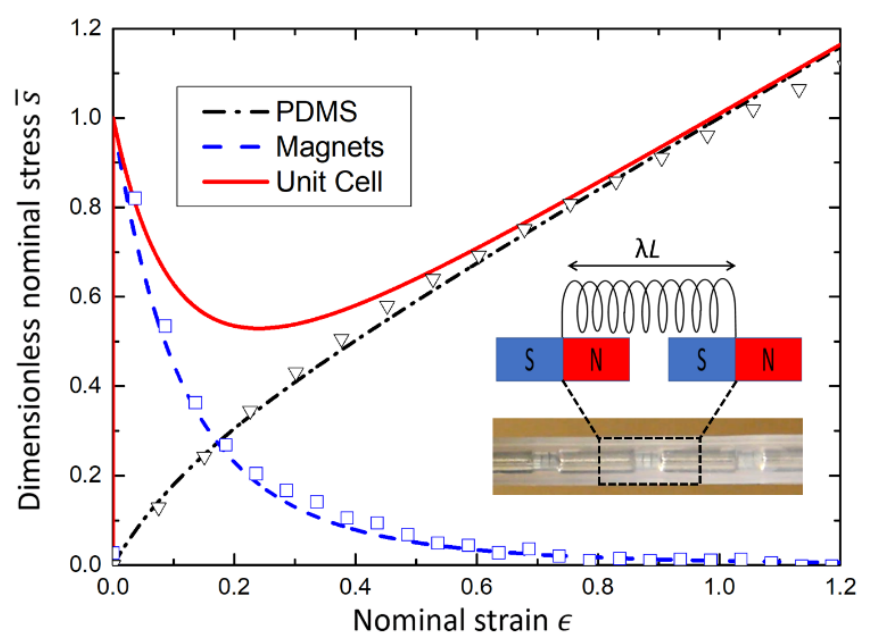

Fig. 5 Dimensionless nominal stress-strain curves of a unit cell ( 26 vol\% magnet) as given by the theoretical model. The material parameters are extracted from independent experiments on the base materials individually. The experimental data for a chain of magnets data are shown by blue squares and those of a pure PDMS sample by black triangles. The inset shows a unit cell and the simplified model.

Here the vacuum permeability $\mu_{0}$ is used to approximate that in the PDMS matrix and in the air gap between magnets.

When the magnets in a unit cell are separated into two groups, a bigger magnet with several spacers on one side and another bigger magnet with the other spacer(s) on the other side. Since only 3 spacers exist between two big magnets, we can simply denote the dipole moment of one magnet and stopper(s) on one side as $m_{1}$, the dipole moment on the other side with the other stopper and leftover stopper(s) as $m_{2}$, with the center-to-center distance $r$ related to the axial stretch $\lambda$ and the undeformed unit-cell length $L$ approximately as $r=L(\lambda-$ $0.5)$. Dividing the magnetic attraction by the undeformed crosssectional area of a unit cell $A$, we obtain the magnetic nominal stress

$$
s_{\mathrm{m}}=\frac{3 \mu_{0} m_{1} m_{2}}{2 \pi A L^{4}\left(\lambda-\frac{1}{2}\right)^{4}}
$$

While more accurate models are available for the attraction between permanent magnets ${ }^{34-36}$, the added mathematical complexity does not necessarily lead to a clearer physical picture for understanding. Here we also neglect the magnetic interactions between non-neighboring magnets. The magnetic nominal stress is always a monotonic decreasing function of the axial stretch, as shown by the dash curve in Fig. 5 .

When a magnet link is disjointed, the surrounding PDMS matrix is stretched elastically, giving rise to an axial tension. For simplicity, we neglect the magnet-matrix interaction (adhesion or friction) and assume a uniform state of deformation with axial stretch $\lambda$ in the matrix. By using an incompressible neoHookean material law with initial shear modulus $\mu$, the elastic nominal stress is evaluated as:

$$
s_{\mathrm{e}}=\mu \frac{A_{\mathrm{p}}}{A}\left(\lambda-\frac{1}{\lambda^{2}}\right)
$$

where $A_{\mathrm{p}}$ is the effective cross-sectional area of the PDMS matrix in a unit cell.

The axial nominal stress in a unit cell of the composite is the sum of the two contributions, $s=s_{\mathrm{e}}+s_{\mathrm{m}}$. In the following discussion, we further normalize all stresses by the magnetic stress in the undeformed state, $s^{*}=24 \mu_{0} m_{1} m_{2} / \pi A L^{4}$, and the dimensionless nominal stress now writes

$$
\bar{s}=\frac{1}{(2 \lambda-1)^{4}}+\alpha\left(\lambda-\frac{1}{\lambda^{2}}\right) .
$$

Here, the dimensionless parameter

$$
\alpha=\frac{\pi \mu A_{\mathrm{p}} L^{4}}{24 \mu_{0} m_{1} m_{2}}
$$

characterizes the significance of the matrix elasticity relative to the magnetic attractions. By fitting the experimental data of the magnets and PDMS individually, we obtain the parameter groups $24 \mu_{0} m_{1} m_{2} / \pi A L^{4}=87 \mathrm{kPa}, \mu=67 \mathrm{kPa}$, and $\alpha=0.57$ for the samples of 26 vol\% magnets.

The dimensionless nominal stresses are plotted as functions of the nominal strain $\epsilon=\lambda-1$ in Fig. 5. The non-monotonic stress-strain curve indicates two equilibrium states under an intermediate dimensionless stress - one corresponds to the state with connected magnets in a unit cell, and the other corresponds to the state of separated magnets (the open state). When a unit cell is loaded beyond $\bar{s}=1$, the magnetic link snaps open dynamically, towards the other equilibrium state at the same stress level. Just like other elastic structures with bistable states, the snaping dissipates energy. Vibration may occur but is expected to be damped out quickly by the matrix viscosity. From the stress-strain curve of a unit cell, it is also evident that the critical stress at which the pair of magnets snap back is much lower, at $\bar{s} \approx 0.5$.

It is noteworthy that the magnets are initially subject to a compressive elastic stress, which balances the magnetic stress (2) in the undeformed state. When an axial tension is applied externally, the magnetic attraction is gradually carried over by the external force, and a minute extension takes place in the magnets as the elastic compression is relieved. However, comparing to the soft PDMS matrix, the magnets are almost rigid and the elastic strains in the magnets is negligible. The elastic part appears as an almost vertical segment on the stressstrain curve of the unit cell, as shown by Fig. 5 .

Let us now move on to the chain level with multiple unit cells connected in series and consider the three stages on the stressstrain curve of a magnetic DN composite. In stage I, all unit cells are in the connected state, and the composite is in a linear elastic regime. If we neglect the stiffness of the PDMS matrix and the change in the magnetic stress $s_{\mathrm{m}}$ at small strains, the stress-strain relation of stage $\mathrm{I}$ is given approximately by

$$
s=\psi_{\mathrm{m}} E \epsilon,
$$

where $\psi_{\mathrm{m}} \approx 1-A / A_{p}$ is the volume fraction of magnets, and $E$ the Young's modulus of the magnets. The separation of one magnetic link ends the deformation in stage I. The choice of the first link to be opened is random and is likely to be caused by imperfections from processing. Nevertheless, at separation, all 
links on the magnetic chain are stretched to the same level, and thus holding similar elastic and magnetic energy. Upon opening of one link, the energy stored in the entire chain will be released and dissipated, similar to the classic Lake-Thomas picture about the rupture of a polymer chain. In contrast to the rupture of a simple elastomer, the energy dissipation does not stop here. Stabilized by the polymer matrix, such a process is to be repeated, which leads to the second stage on the stress-strain curve.

In stage II, some of the magnetic links are in the open state while the rest are still connected. Consider the case when there are $N$ units in total and $M$ of them are open. Neglecting the strain from elastically deforming the connected magnetic links, the overall stretch of the composite, $\bar{\lambda}$ is related to the local stretch $\lambda$ in the open units by

$$
\bar{\lambda}=\frac{M \lambda L+(N-M) L}{N L}=1+\frac{M}{N}(\lambda-1) .
$$

By substituting Eq. (7) into Eq. (4), we arrive at the stressstretch relation in all these states, as shown by the dash curves in Fig. 6.

Throughout a loading process, the axial stress is bounded by the peak value when the magnetic link separates in a single unit cell, $\bar{s}=1$. When further stretched, one of the connected magnetic links snaps open, and the loading curve will jump to the next branch. Maximum magnetic stress between two magnets, $f_{0}$, beyond which an extra pair of magnets will be separated, is the point when the loading curve deescalate to the next one. In this paper, we neglect the dynamics and vibration of the system, and limit the discussion to the quasi-static cases of displacement-controlled loading, so that the jump occurs as a steep drop in force at constant elongation, as shown by the solid red curve in Fig. 6 . Such a process happens repeatedly during loading, until all magnet links are open. This process corresponds to a plateau with repeated ramps and drops on the

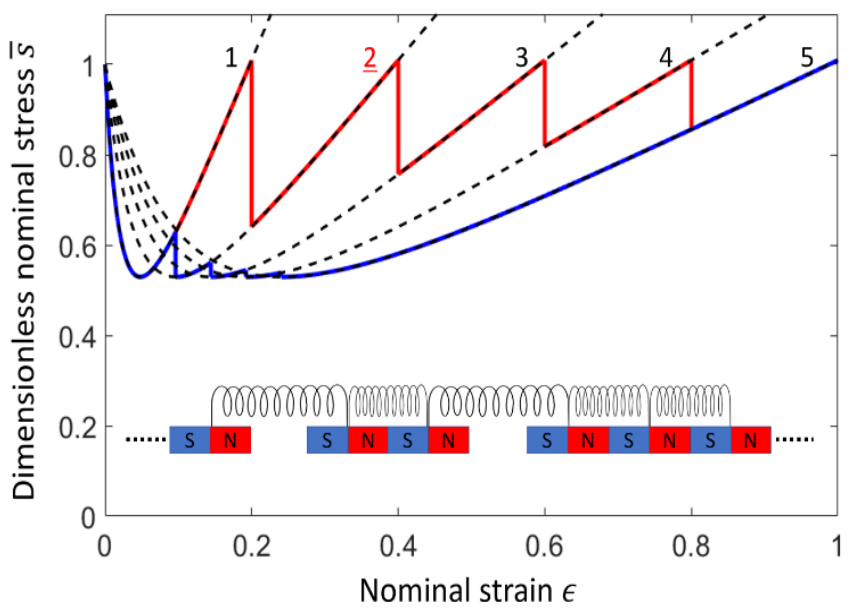

Fig. 6 Theoretical stress-strain curves (dash curves) of a DN composite for the cases containing the indicated numbers of open links. Stress-strain curve (solid red) during loading. One of the magnetic links snap open at the critical stress $\bar{s}=1$, after which the axial stress drops to the next branch with one more link open. Stress-strain curve (solid blue) during unloading. One of the magnetic links snap close at the trough point on each branch, and the stress jumps up to the branch with one less link open. Displacement-controlled loading is assumed when constructing the loading/unloading curves. The sketch in the inset indicates the state in which two links are open. loading curves of the composite. If all magnets are identical, the peak force of the ramps $f_{0}$ remains constant - a gradual increase in the peak force was observed in experiments, possibly due to the nonuniformity of the magnets or the composite structure.

The composite follows a different stress-stretch curve when it is unloaded. All separated unit cells will be relaxed uniformly until the trough of each branch is reached, and then one pair of magnets will snap to contact, and the curve jumps up to the next branch to the left (corresponding to the state of one less open unit), as shown by the solid red curve on Fig. 6 . As the snaping takes place on the troughs of the curve, the stretch is also much lower than that of the separation during loading. That is, the force needed to separate two magnets is much higher than the force at which two far separated magnets snap back. Such an asymmetry between loading and unloading results in a hysteresis enclosed by the loading and unloading curves. This is a major mechanism for energy dissipation in the magnetic DN composite, as well as other structures and material systems with similar long-range forces that decrease with separation, e.g. electrostatic interaction.

If we look closely on one specific unit cell of magnets during the unloading process, we will find that three different damping mechanisms exist and work coherently in the DN magnetic composite. Firstly, the vibration on the magnet itself, which is true for a single chain of magnets even without any matrix material. The kinetic energy during retracting of each magnets will be dissipated in this way. Secondly, the sliding between the PDMS matrix and magnets, in which a specific pair of magnets snapping together, the sliding contributes as a major mechanism to redistribute the load between the magnets and surrounding PDMS matrix, thus making the stress less to close an unit cell compared with the stress to open an unit cell. Thirdly, the vibration of the PDMS matrix itself. After one open unit cell is snapped back, the surrounding PDMS initiates a wave propagating from the center of the unit cell to both directions, corresponding to a dynamic damping effect. The troughs throughout the unloading curve are at a constant level in the ideal case. In experiments, these stress values of the troughs decrease gradually during unloading, as shown by Fig. 4 . The ramps and jumps on both the loading and unloading curves are caused by the discrete nature of the structure, and the overall smoothness is dependent on size of the system or the number of unit cells.

The model prediction is presented in a dimensionless form, and the dependences on material parameters are all included through the normalization, e.g. via the magnetic stress $s^{*}=$ $24 \mu_{0} m_{1} m_{2} / \pi A L^{4}$. To make a direct comparison between the theoretical model and experiments, let us look at the composition dependence of the peak nominal stress, by inserting independently measured parameters. Keeping the same number of magnet chains, and varying the PDMS content, we modify the magnet volume fraction $\psi$. The theory predicts a linear relation between the peak stress and the volume fraction: $s^{*} \propto \psi$, which agrees well with the experimental results as shown by Fig.7. In the same figure, the lower plateau stress on the unloading curve predicted by the model is also plotted against experimental data. Although showing the same 


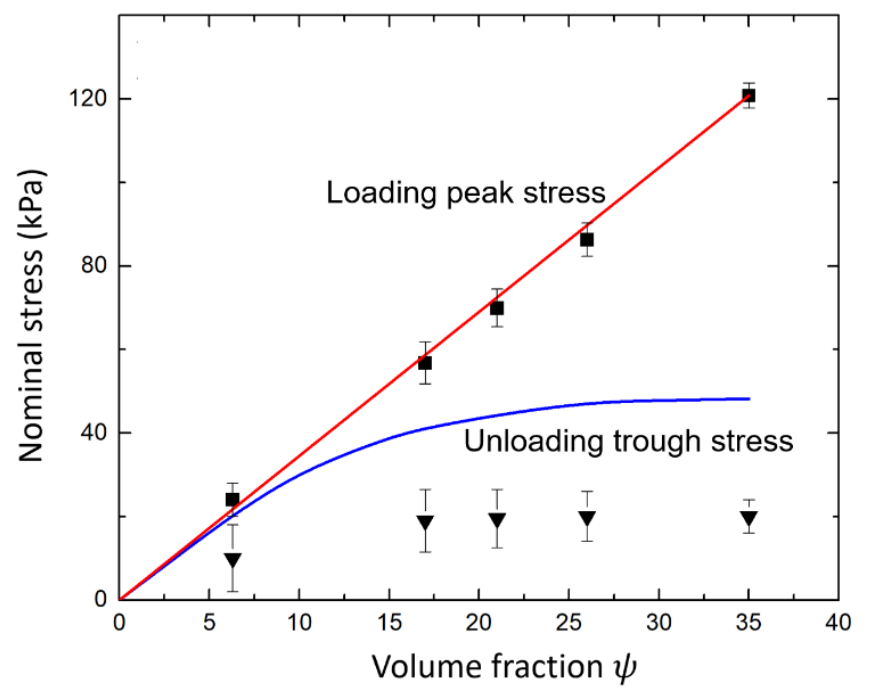

Fig. 7 The loading peak stress and unloading trough stress of the magnetic DN composite, plotted as functions of the magnet volume fraction $\psi$. The solid curves are the theoretical prediction of the simple frictionless model, and the solid symbols show the experimental data.

trend, the experiments exhibit significantly lower values than the theory prediction, giving a much larger hysteresis. The difference may be attributed to the effect of sliding friction or adhesion between the PDMS matrix and the magnets, as will be discussed in the following section.

\section{Discussion}

In general, the nominal stress-strain curve of a unit cell of the composite is non-monotonic, containing three segments: the first part is an initially linearly increasing segment with a very large slope close to the rigidity of the magnets; the second part is the decreasing segment dominated by the declining magnetic attraction; and the third part is the increasing segment at large separation, dominated by the elastic force from the matrix. The minimum axial force marks the separation point of the second and third region. When the unit cell is in a composite of multiple unit cells in serial, the second part is unstable. When loaded beyond the peak of the first part, the unit cells will transit from all-contact states to all-separated states in a random sequence. After reaching all-separated states, the axial force is mainly carried by the PDMS matrix.

The measured loading curves differ slightly from the prediction of the simple model, while the difference is more pronounced between unloading curves. The loading curve stands on top of the theoretical curve while the unloading curve exhibits much lower stress than that given by the simple model. We attribute these differences to the effects of interfacial adhesion and/or friction. Consider the force transducing between the magnet and the matrix in a unit cell, as illustrated by Fig. 8 . In the case of a frictionless interface, the axial forces in the matrix and the magnet are both uniform, as shown by the dash lines in Fig. 8. When friction of adhesion is present, the axial force is gradually transferred between the magnet and the matrix by friction/shear force. The friction/shear force are acting towards opposite directions during loading and unloading. During loading, the friction induces higher tension in the matrix segment between two separated magnets, so that the local strain and thus stress in the matrix is higher than the uniform case. During unloading, the friction/shear force is
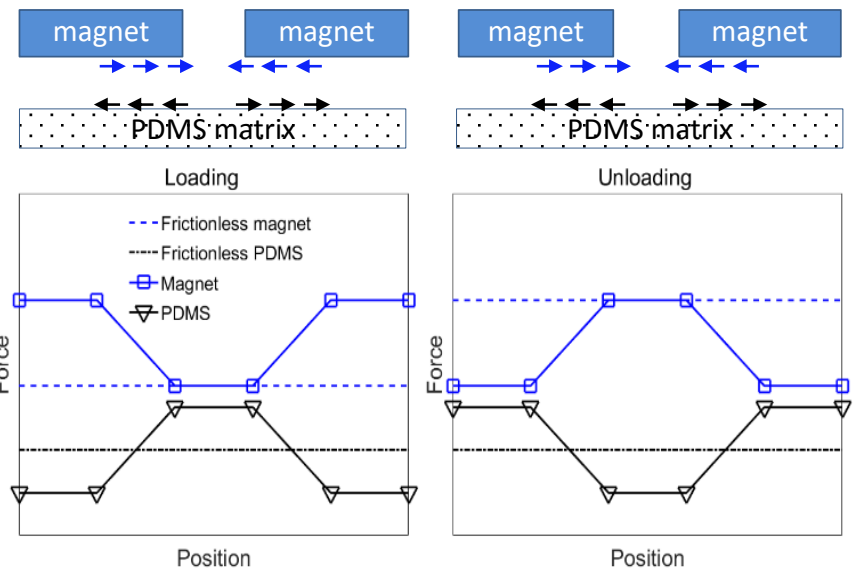

Fig. 8 Sketch illustrating the interaction between the PDMS matrix and the magnets, during loading and unloading processes.

compressing the middle matrix segment, causing a lower stress than the uniform/frictionless case.

Using a simple shear-lag model, ${ }^{27}$ and assuming constant shear stress $\tau$ at the interface, we calculate the nominal axial stress of a unit cell due to elastic deformation, and plot it with dash lines in Fig. 9, for both loading and unloading cases. By adding the same magnetic contribution as in Eq. (4), we obtain the total nominal stress in a unit cell, as shown by the solid curves in Fig. 9. A dimensionless parameter describing the magnitude of the friction/shear stress, $2 \pi r \tau / A_{p} \mu$, with $r$ being the radius of the magnet, is taken to be 0.01 when plotting Fig. 9. Comparing to the frictionless case (dotted curves on Fig. 9), even though the same shear-lag stress is taken for both loading and unloading cases, the effect is more significant in decreasing the axial stress during unloading, just as observed in the experiments. The simple shear-lag model, however, is inapplicable to the transition stage between loading and unloading, when the shear forces will switch direction and the distribution is by no means uniform.

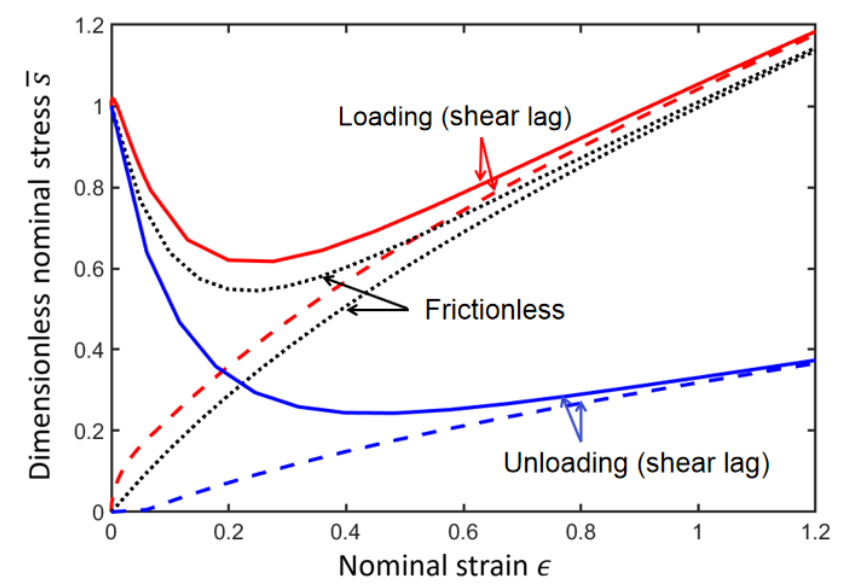

Fig. 9 Dimensionless nominal stress-strain curves of a unit cell when considering the effect of interfacial friction. The solid curves include the magnetic attractions while the dash curves do not. The friction is modelled by a shear-lag model of constant shear stress $\tau$ at the place of sliding. The dimensionless friction $2 \pi r \tau / A_{p} \mu$ is taken to be 0.01 for both loading and unloading. The frictionless limit is plotted with dotted curves as a reference. 


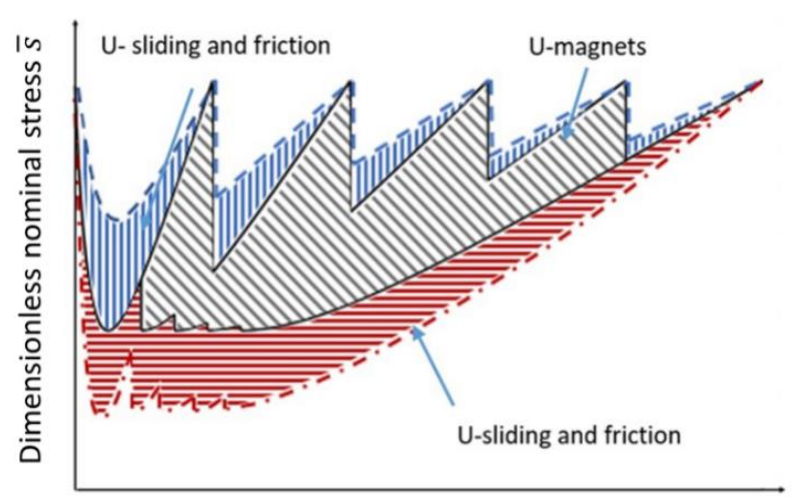

Nominal strain $\epsilon$

Fig. 10 Schematic drawing of loading and unloading curves (dash curves) relative to the theoretical prediction of the ideal frictionless case (solid curve).

A complete stress-strain curve of multiple unit cells connected in serial may be constructed by populating the unitcell curves of the shear-lag model, and switching among different branches when critical points are reached during loading and unloading, following the same approach of constructing Fig. 6. However, due to the lack of necessary parameters and the inapplicability of the shear-lag model at the transition stage, here we schematically draw the loading and unloading curves in Fig. 10, together with the theoretical prediction of the ideal frictionless case. The actual loading curve is always above the ideal case while the unloading curve is below. A larger hysteresis is expected due to the energy dissipation from the sliding against friction/adhesion.

As the interfacial friction/adhesion plays such an important role in energy dissipation and thus toughening, we experimented a similar design by using a commercial adhesive tape, 3M VHB 4910, as the matrix. Each train of magnets are fabricated by rolling the magnets inside a layer of the VHB tape, as illustrated by Fig. 11. The strong interfacial adhesion is expected to significantly enhance the energy dissipation. The mechanical tests were carried out with a same setup as described above.

The VHB-based composite also exhibited a very similar toughening mechanism, with a representative force-stretch curve shown in Fig.12. The saw teeth on the plateau of the curve were also seen to be shallower due to the stronger adhesion/friction at the interface. We built a 2D composite by laying the chains side by side, with a half-period offset between neighboring chains, as shown by Fig. 13. A typical stress-strain curve of a sample with five parallel chains is shown in Fig. 13.

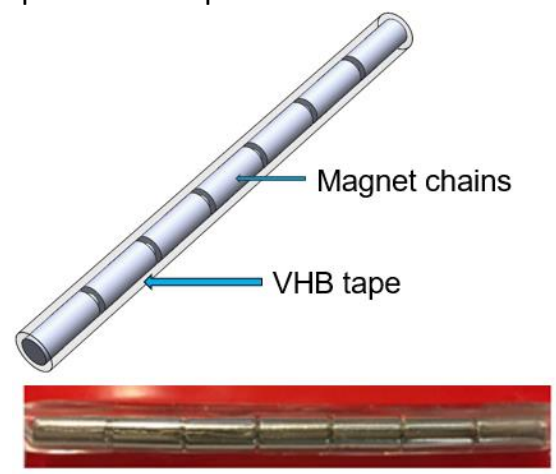

Fig. 11 Schematic of the fabrication process of a VHB-based composite (top) and photograph of a single-chain composite (bottom).

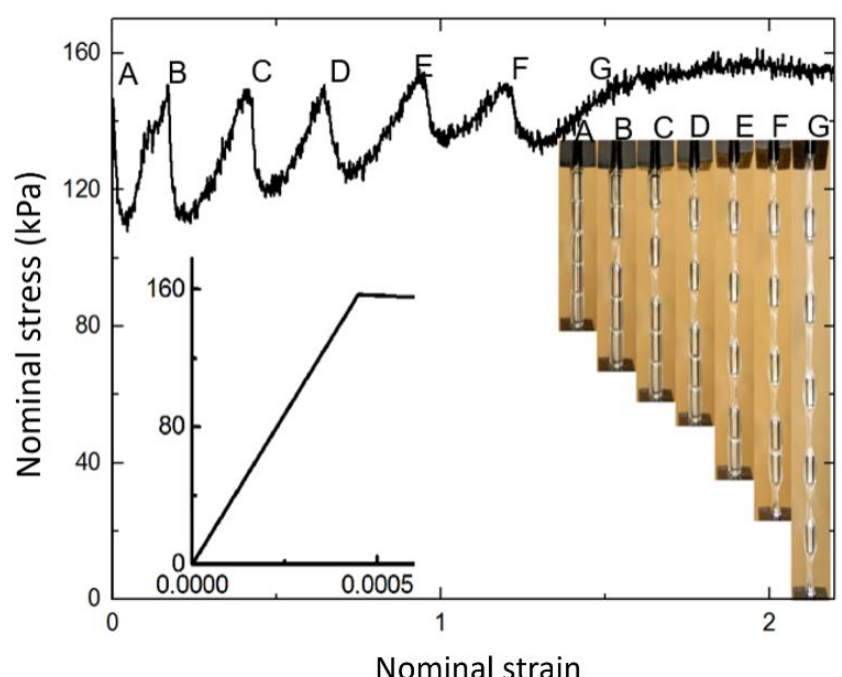

Fig. 12 Nominal stress-strain curves of a VHB-based magnetic DN composite. The insets A-G are the snapshots of the sample corresponding to the specific points along the loading curves. Over the stress plateau, partially damaged regions coexisted with the undamaged regions, and the extension was accompanied by the growth of the "damaged" regions. The initial region curves with an exaggerated strain scale shown at bottom left.

Due to the synergetic effect among different chains, which were misaligned deliberately, the saw teeth were smoothed out in a 2D composite. Comparing to the PDMS-based composite, the toughness of the VHB-based composite was evidently enhanced, although the underlying mechanism remained unchanged.

\section{Conclusion}

In this work, we designed and tested a magnetic DN composite capable of large and recoverable deformation. In the composite, initially connected chains of permanent magnets provided relatively high stiffness, served as the sacrificial components for energy dissipation, and could spontaneously reconnect upon unloading. Exhibiting a stress plateau over the

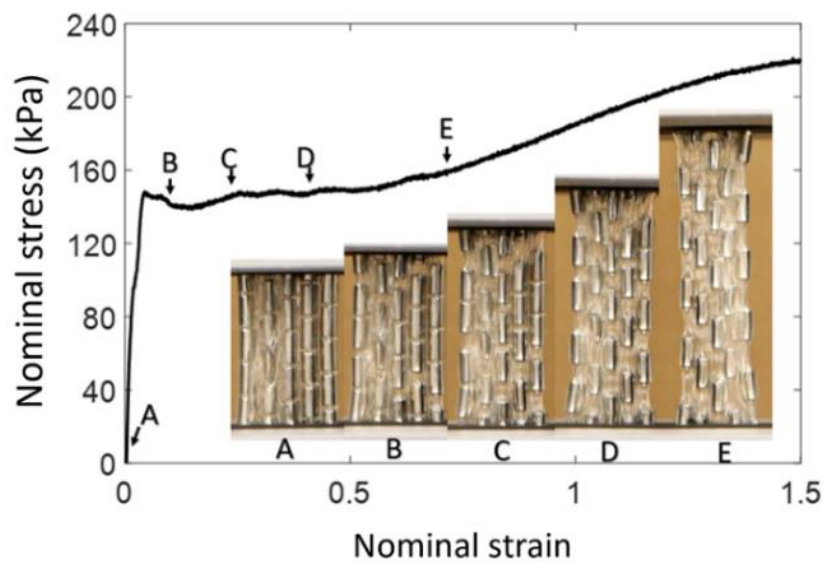

Fig. 13 Nominal stress-strain curves of a VHB-based magnetic DN composite. The insets $A-E$ are the snapshots corresponding to the specific points along the loading curves. The curve is smoothed by the synergetic effect among multiple chains. Over the stress plateau (B-E), partial damage regions (magnets separated) coexisted with the undamaged regions, and the extension was accompanied by the growth of the damage regions. In the strain-hardening state, all magnets were separated, and the extension caused further sliding between magnets and VHB matrix. 
loading curve of tensile test, the damage evolution process was similar to that of DN gels and elastomers. The strength of the composite was limited by the magnetic attractions, while the maximum stretch was limited by the stretchability of the matrix. The composite was significantly tougher than either of the constituents. Upon unloading, the magnetic attraction between neighboring magnetics held them back again and the composite's stiffness and strength were fully recovered. By combining the properties of high-stretchability, self-healing, and high-toughness, the DN composite is a viable candidate for many applications. Overall, the magnetic composite can be treated as a microscopic model of tough physical hydrogels composed of reconnecting bonds. ${ }^{18}$ To capture the damage evolution process and to better understand the toughening mechanism, a simple theoretical model is introduced to study the damage distribution and energy dissipation process. The model predicts the stress-strain relation of the magnetic DN composite. Through visualizing the energy dissipation process, it may provide insights towards further understanding of the toughening mechanism and optimizing magnetic DN composites for enhanced properties.

\section{Acknowledgments}

WH acknowledges the Bailey Research Career Award from lowa State University, and the financial support by the National Natural Science Foundation of China through Grant 11972015.

\section{Notes and references}

1 J. P. Gong, Y. Katsuyama, T. Kurokawa and Y. Osada, Adv. Mater., 2003, 15, 1155.

2 J. P. Gong, Soft Matter, 2010, 6, 2583.

3 A. Nakayama, A. Kakugo, J. P. Gong, Y. Osada, M., Takai, and T. Erata, Adv. Funct. Mater., 2004, 14, 1124.

4 K. Yasuda, J. P. Gong, Y. Katsuyama, A. Nakayama, Y. Tanabe, E. Kondo, M. Ueno, and Y. Osada, Y., Biomaterials, 2005, 26, 4468.

5 Illeperuma, W. R., Sun, J. Y., Suo, Z., \& Vlassak, J. J. (2014). Fiber-reinforced tough hydrogels. Extreme Mechanics Letters, 1, 90-96.

6 Reichert, W. F., Göritz, D., \& Duschl, E. J. (1993). The double network, a model describing filled elastomers. Polymer, 34(6), 1216-1221.

7 Santangelo, P. G., \& Roland, C. M. (1994). The mechanical behavior of double network elastomers. Rubber chemistry and technology, 67(2), 359-365.

8 Ducrot, E., Chen, Y., Bulters, M., Sijbesma, R. P., \& Creton, C. (2014). Toughening elastomers with sacrificial bonds and watching them break. Science, 344(6180), 186-189.

9 Sun, J. Y., Zhao, X., Illeperuma, W. R., Chaudhuri, O., Oh, K. H., Mooney, D. J., ... \& Suo, Z. (2012). Highly stretchable and tough hydrogels. Nature, 489(7414), 133-136.

10 C. Azuma, K. Yasuda, Y. Tanabe, H. Taniguro, F. Kanaya, A. Nakayama, Y. M. Chen, J. P. Gong, and Y. Osada, J. Biomed. Mater. Res. A, 2007, 81, 373.

11 M. A. Haque, T. Kurokawa, and J. P. Gong, Polymer, 2012, 53, 1805.

12 H. Tsukeshiba, M. Huang, Y. H. Na, T. Kurokawa, R. Kuwabara, Y. Tanaka, H. Furukawa, H., Y. Osada, and J. P. Gong, J. Phys. Chem. B, 2005, 109, 16304.
13 B. J. DeKosky, N. H. Dormer, G. C. Ingalve, C. H. Roatch, J. Lomakin, M. S. Detamore and S. H. Gehrke, Tissue Eng. C, 2010, 16, 1533.

14 A. Rakovsky, D. Marbach, N. Lotan and Y. Lanir, J. Appl. Polym. Sci., 2009, 112, 390.

15 Bao, G., and Z. Suo. "Remarks on crack-bridging concepts." (1992): 355-366.

16 King, Daniel R., et al. "Extremely tough composites from fabric reinforced polyampholyte hydrogels." Materials horizons 2.6 (2015): 584-591.

17 Lin, Shaoting, et al. "Design of stiff, tough and stretchy hydrogel composites via nanoscale hybrid crosslinking and macroscale fiber reinforcement." Soft matter 10.38 (2014): 7519-7527.

18 Cooper, Christopher B., et al. "Toughening stretchable fibers via serial fracturing of a metallic core." Science advances 5.2 (2019): eaat4600.

$19 \mathrm{Li}$, Chenghai, et al. "Fatigue-resistant elastomers." Journal of the Mechanics and Physics of Solids 134 (2020): 103751.

20 Xiang, Chunping, et al. "Stretchable and fatigue-resistant materials." Materials Today 34 (2020): 7-16.

21 Zhu, Fengbo, et al. "3D-printed ultratough hydrogel structures with titin-like domains." ACS Applied Materials \& Interfaces 9.13 (2017): 11363-11367.

22 Gong, J. P. (2014). Materials both tough and soft. Science, 344(6180), 161-162.

23 Keplinger, C., Sun, J. Y., Foo, C. C., Rothemund, P., Whitesides, G. M., \& Suo, Z. (2013). Stretchable, transparent, ionic conductors. Science, 341(6149), 984-987.

24 Sun, T. L., Luo, F., Kurokawa, T., Karobi, S. N., Nakajima, T., \& Gong, J. P. (2015). Molecular structure of self-healing polyampholyte hydrogels analyzed from tensile behaviors. Soft matter, 11(48), 9355-9366.

25 Sun, T. L., Kurokawa, T., Kuroda, S., Ihsan, A. B., Akasaki, T., Sato, K., ... \& Gong, J. P. (2013). Physical hydrogels composed of polyampholytes demonstrate high toughness and viscoelasticity. Nature materials, 12(10), 932-937.

26 Zhao, X. (2014). Multi-scale multi-mechanism design of tough hydrogels: building dissipation into stretchy networks. Soft Matter, 10(5), 672-687.

27 Feng, X., Ma, Z., MacArthur, J. V., Giuffre, C. J., Bastawros, A. F., \& Hong, W. (2016). A highly stretchable double-network composite. Soft matter, 12(44), 8999-9006.

28 Dudek, K. K., Gatt, R., \& Grima, J. N. (2020). 3D composite metamaterial with magnetic inclusions exhibiting negative stiffness and auxetic behaviour. Materials \& Design, 187, 108403.

29 Dudek, K. K., Wolak, W., Gatt, R., \& Grima, J. N. (2019). Impact resistance of composite magnetic metamaterials. Scientific reports, 9(1), 1-9.

30 Grima, J. N., Caruana-Gauci, R., Dudek, M. R., Wojciechowski, K. W., \& Gatt, R. (2013). Smart metamaterials with tunable auxetic and other properties. Smart Materials and Structures, 22(8), 084016.

31 Fang, H., Chang, T. S., \& Wang, K. W. (2019). Magneto-origami structures: Engineering multi-stability and dynamics via magnetic-elastic coupling. Smart Materials and Structures, 29(1), 015026.

32 Slesarenko, V. (2020). Planar Mechanical Metamaterials with Embedded Permanent Magnets. Materials, 13(6), 1313.

33 Guell Izard, A., \& Valdevit, L. (2020). Magnetoelastic metamaterials for energy dissipation and wave filtering. Advanced Engineering Materials, 22(2), 1901019.

34 J.S. Agashe, D.P. Arnold, J. Phys. D: Appl. Phys. 41 (2008) 105001.

35 Vokoun, D., Beleggia, M., Heller, L., \& Šittner, P. (2009). Magnetostatic interactions and forces between cylindrical permanent magnets. Journal of magnetism and Magnetic Materials, 321(22), 3758-3763. 
36 Tang, X., Chen, Y., \& Conrad, H. (1996). Structure and interaction force in a model magnetorheological system. Journal of intelligent material systems and structures, 7(5), 517-521. 